\title{
Droplet Dynamics and Heat Transfer on Wettability Patterned Surfaces
}

\author{
Xin Cai, Minghao He, Huihe Qiu* \\ Department of Mechanical and Aerospace Engineering \\ The Hong Kong University of Science \& Technology \\ Hong Kong SAR, China \\ "meqiu@ust.hk
}

\section{Extended Abstract}

The shape of liquid droplets on chemically structured surfaces is determined by the interplay of the interfacial free energies of the droplet and the wettability pattern on the surface for micro droplets. This interplay can give rise to morphological transitions upon changing the volume of the droplet or the wettability contrast and thus the contact angle of the substrate [1,2]. Morphological transitions of single droplets have been theoretically and experimentally studied for a variety of wettability patterns. Morphological transitions take place if the droplet volume becomes comparable or bigger than a reference volume defined by the surface domain. The dynamic behaviour of a liquid droplet on a solid heterogeneous surface is extremely rich and plays an important role in academic and industrial communities, such as coating, printing, or microfluidics. Though significant progress has been made in this area, many unanswered questions remain. A large number of experimental, theoretical, and numerical studies have been performed by many scientists. Kusumaatmaja et al. [1-5] found that the initial drop conditions have a deep influence on the final shape of the droplet when the droplet typically wets to three strips, and they proposed a way that using chemically patterned surfaces to manipulate the behaviour of micron-scale drops. Chang et al. [6] studied the spreading behaviour of small droplets on up to three hydrophobic and hydrophilic strips, they found the phenomena that the spreading behaviour depends on the width of the hydrophobic strip, the initial location, and gravity. The study of spreading dynamics of droplets on patterned surfaces is very attractive, however considering the large cost and difficulties of the experimental work on micro and mesoscopic scale, numerical modelling can be very useful for studying liquid drops on solid surfaces of complex structure and chemistry. The lattice Boltzmann method (LBM) has a strength which is the ability to simulate multi-component multiphase fluids [7]. For simulating multiphase fluid flows, Shan \& Chen [8] have initiated a pseudo-potential model which is a very popular multiphase LBM model and is better defined in terms of interparticle potential and the liquid-solid interactions.

In this paper, droplet dynamics on wettability patterned surfaces is investigated. Critical stages of droplets on hydrophilic dot of hydrophobic surfaces were studied experimentally and numerically. The critical droplet size and critical contact angle were measured and compared with numerical analysis. To study droplet dynamics and sliding on wettability patterned surfaces, a three-dimensional model has been developed by using Shan-Chen type multiphase lattice Boltzmann method (LBM). On the solid heterogeneous wall, a hydrophobic strip is positioned in the centre of the lattice, followed by a hydrophilic strip on both sides of this strip. We investigate the effects of different initial conditions on the spreading behaviour and final shape of the droplet. These factors including: the initial location of the droplet, the relative widths of the strips, and the radius of the droplet. A dimensionless linear relationship between these initial conditions and droplet spreading behaviour has been proposed and it is able to predict droplet behaviour as a function of surface structure and droplet properties using numerical modelling. We also systematically study the evaporation of multicomponent droplet on heated wettability patterned surface comparing to homogenous surface. The results of evaporation duration show that wettability patterned surface can enhance evaporation by elongating contact line.

\section{References}

[1] A. Darhuber, S. M. Troian, S. M. Miller and S. Wagner, "Morphology of liquid microstructures on chemically patterned surfaces," Appl. Phys., vol. 87, pp. 7768-7775, 2000. 
[2] M. Brinkmann and R. Lipowsky, "Wetting morphologies on substrates with striped surface domains," Appl. Phys., vol. 92, pp. 4296-4306, 2002.

[3] Dupuis and J. M. Yeomans, "Lattice Boltzmann modelling of droplets on chemically heterogeneous surfaces," Fut. Gen. Comp. Syst., vol. 20, no. 6, pp. 993-1001, 2004.

[4] H. Kusumaatmaja, J. L’eopold`es, A. Dupuis and J. M. Yeomans, Drop dynamics on chemically patterned surfaces. Europhys. Lett., 2006, 73 (5): 740-746.

[5] H. Kusumaatmaja* and J. M. Yeomans, "Controlling Drop Size and Polydispersity Using Chemically Patterned Surfaces," Langmuir, vol. 23, pp. 956-959, 2003.

[6] Q. Chang and J. I. D. Alexander, "Analysis of single droplet dynamics on striped surface domains using a lattice Boltzmann method," Micro d גiano घ, wiol. 2, pp. 309-326, 2006.

[7] S. Gong and P. Cheng*, "Numerical investigation of droplet motion and coalescence by an improved lattice Boltzmann model for phase transitions and multiphase flows," Computers \& Fluids, vol. 53, pp. 93-104, 2012.

[8] X. Shan and H. Chen, "Lattice Boltzmann model for simulating flows with multiple phases and components," Phys. Rev. E., vol. 47, no. 3, pp. 1815, 1993. 